\title{
Analysis of cross-program effects in the sphere of small and medium-sized businesses in the region (on the example of the Kurgan region)
}

\author{
Tatiana Khudyakova ${ }^{1, *}$ \\ ${ }^{1}$ Ural State University of Economics, 620144, 62, 8 Marta st., Ekaterinburg, Russia
}

\begin{abstract}
The article is devoted to the identification and analysis of crossprogram effects in the sphere of small and medium-sized businesses that are formed during the implementation of state programs of the Kurgan region. The relevance and usefulness of identifying inter-program connections are proved. The state programs of the Kurgan region that form the effects in the sphere of small and medium-sized businesses in the region are identified. Groups of cross-program effects were formed and the number of state programs and their activities that form various types of effects in the sphere of small and medium-sized businesses in the region was found. The assessment of cross-program effects for each task of the program of small and medium-sized businesses development in the region was carried out. The paper presents quantitative results of assessment of cross-program effects value created by state programs for each group of cross-program effects and each task of the program for the development of small and medium-sized businesses in the region.
\end{abstract}

\section{Introduction}

State programs dedicated to various areas of socio-economic development, including support for small and medium-sized businesses (hereinafter referred to as SMB), are implemented in all regions of the Russian Federation. The effects obtained in a particular social and economic sphere are made up of the results of the implementation of not only a single state program. But also they are the result of the influence of other state programs implemented in the same region. The author suggests to name such effects as crossprogram effects (hereinafter - CPEs), which issues of identification and assessment are practically not studied. This article attempts to identify and analyze inter-program connections that form CPEs in the SMB sector on the example of the Kurgan region.

The relevance of inter-program relationships identification and analysis is noted in the guidelines for the development and implementation of state programs of the Russian Federation, approved by the order of the Ministry of Economic Development of the Russian Federation No. 582 from September 16, 2016. This document emphasizes the need to identify links between the activities of some state programs and the goals and objectives of other state programs. Also, the consolidated annual report of the Ministry of Economic Development of the Russian Federation on the implementation and assessment of state

\footnotetext{
*Corresponding author: khudyakova_t@mail.ru
} 
programs of the Russian Federation effectiveness in 2017 also states the need and intention of the Ministry to work on the formation of a system for assessing the cross-impact of state programs [1].

In the scientific environment, there are also prerequisites for analyzing inter-program connections. Authors S.N. Rukin and V.N. Samodurova note that when analyzing the effectiveness of budget expenditures, it is necessary to take into account the assessment of the entire set of financial, administrative and other resources [2]. In studies of A.G. Breusova appropriateness of study of the possibilities of reducing government programs costs without losing results and quality is pointed out [3]. N.S. Shmigol emphasizes the usefulness of comparing state programs with each other in order to periodically re-evaluate the functions and tasks assigned to federal executive bodies in order to identify outdated, duplicative and ineffective programs. [4] Moreover, the first attempts to identify interprogram connections were made several years ago by the research team of the Institute of Macroeconomic Research [5]. N.N. Shash also emphasizes the importance of taking into account the indirect effects of state programs implementation that relate to other areas of social and economic development [6]. The issues of cross-influence and interdependence of various elements within the same social and economic system are also addressed in the works of some foreign authors [7,8]. Based on the results of previous studies, the author is of the opinion that the relationship between the activities of one state program and the goals/objectives of another state program is involved in the CPEs formation [9].

\section{Materials and methods}

In the course of the research, regulatory, comparative, and computational methods, content analysis, and the method of pairwise comparison were applied. The regulatory method was used to analyze regulatory documents containing state programs in order to study the social and economic orientation and program activities of state programs. The comparative method was used to compare the goals, tasks, and program activities of state programs in order to identify state programs that have an impact on the same area of social and economic development. Content analysis was used to study the texts of program documents in order to identify logical links between government programs. Pairwise comparison and calculation methods were used to determine the value of the CPEs generated by the programs for each group of CPEs and each task of the SMB development program in the region.

The initial information base for the analysis was the regulatory documents approving the state programs of the Kurgan region, published on the official websites of the government of the Kurgan region and responsible executives of state programs.

At the first stage of the study the key state program of the region dedicated to the development of SMB, and other state programs were identified, which activities contribute to the achievement of the goals and objectives of the key state program. At the second stage of the study, all activities of state programs were distributed among pre-formed groups of CPEs and the most expressed group of CPEs was identified. At the third stage of the study, using pairwise comparisons of all previously identified groups of measures that create CPEs in the SMB sector, their priority vectors (significance coefficients) were determined relative to each task of the key state program dedicated to SMB development. Then, at the fourth stage of the study, the value of the CPEs manifestation for each group of crossprogram effects in relation to a specific task of the key state program was determined and the total CPEs manifestation for each task of the key state program was calculated. At the final stage of the study, conclusions were drawn about the participation of other state programs in the region in achieving the goals and forming the effects of the SMB development program in the region. 


\section{Results and Discussion}

The key state program dedicated to the development of SMB in the Kurgan region is the state program "On the development and support of small and medium-sized businesses in the Kurgan region" for 2014-2020. Content analysis of other state programs activities in the Kurgan region showed that 17 out of 45 state programs (38\%) contain activities that affect the development of SMB in the region. All identified activities were classified into the following groups that create the effects of SMB development: financial, sales, innovation, infrastructure, supervisory and licensing, information and consulting, and others.

The number of government programs that affect the SMB sector in the Kurgan region, with the exception of the key program, is shown in the figure.

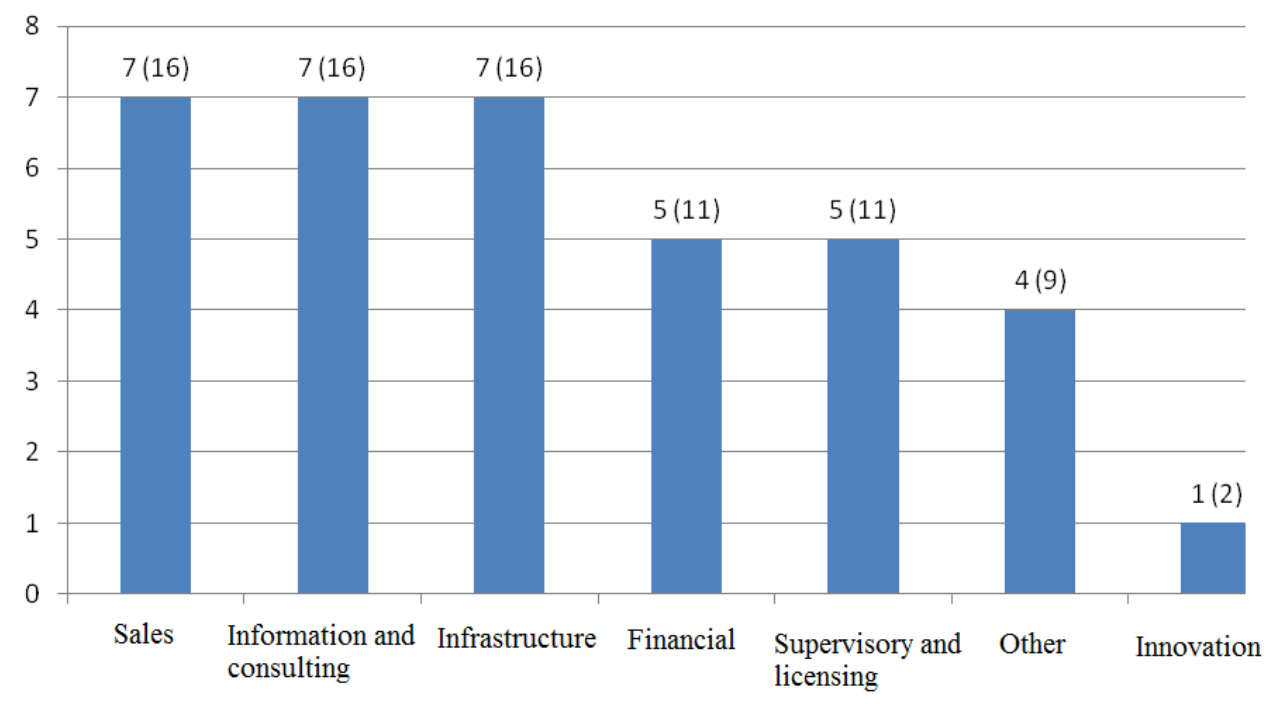

Fig. 1. The number of other state programs of the Kurgan region that form cross-program effects in the sphere of small and medium-sized businesses in the region, pcs. (\%).

The most measures affecting the development of SMB in the Kurgan region contain the state program "Development of the agro-industrial complex in the Kurgan region for 20142020 " (23.5\% of measures). The remaining state programs contain two or more times fewer activities that affect SMB development. However, among other government programs number of measures contributing to SMB development in the Kurgan region are highlighted in the state program "Tourism development in the Kurgan region", "Development of industry and increasing its competitiveness", "Improving conditions and labor protection in the Kurgan region" (11\% of measures), "Development of science and technologies for the period till 2020" and the state program of Kurgan region aimed at creation of favorable conditions for attracting investments into the economy of the Kurgan region ( $8 \%$ of measures). According to the coverage of CPEs groups, the state programs "Development of industry and increasing its competitiveness" (86\% of groups) and the state program of the Kurgan region aimed at creating favorable conditions for attracting investment in the economy of the Kurgan region ( $71 \%$ of groups) are allocated, the remaining programs cover from $14 \%$ to $43 \%$ of CPEs groups.

The results of the final CPEs assessment for each task of the key state program for SMB development in the Kurgan region are presented in the table. 
Table 1. Conditional manifestation of cross-program effects of state programs that affect the objectives of SMB development program of the Kurgan region.

\begin{tabular}{|c|c|c|c|c|c|c|c|c|c|c|}
\hline \multirow{2}{*}{\multicolumn{2}{|c|}{$\begin{array}{l}\text { Groups of cross- } \\
\text { program effects in } \\
\text { SMB sector }\end{array}$}} & \multicolumn{7}{|c|}{ Objectives of SMB development program } & \multicolumn{2}{|c|}{$\begin{array}{c}\text { Final CPEs } \\
\text { manifestatio } \\
\mathrm{n}\end{array}$} \\
\hline & & 1 & 2 & 3 & 4 & 5 & 6 & 7 & c.u. & $\%$ \\
\hline \multicolumn{2}{|l|}{ 1. Financial } & - & 5.98 & 16.08 & 14.16 & 6.12 & - & 0.72 & 43.06 & 71.1 \\
\hline \multicolumn{2}{|l|}{ 2. Sales } & - & 0.88 & - & - & 2.07 & - & 0.26 & 3.21 & 5.3 \\
\hline \multicolumn{2}{|c|}{$\begin{array}{l}\text { 3. Supervision and } \\
\text { licensing }\end{array}$} & - & 0.02 & - & 0.05 & 0.09 & - & 0.06 & 0.22 & 0.4 \\
\hline \multicolumn{2}{|c|}{$\begin{array}{l}\text { 4. Information and } \\
\text { consulting }\end{array}$} & - & 1.30 & 0.06 & 0.80 & 1.12 & 2.84 & 0.14 & 6.26 & 10.3 \\
\hline \multicolumn{2}{|l|}{ 5. Innovative } & - & 0.28 & 0.05 & 0.03 & 0.32 & 0.42 & 1.88 & 2.98 & 4.9 \\
\hline \multicolumn{2}{|c|}{ 6. Infrastructure } & - & 2.31 & 0.66 & 0.68 & 0.98 & 0.08 & 0.07 & 4.78 & 7.9 \\
\hline \multicolumn{2}{|l|}{ 7. Other } & - & - & - & - & 0.04 & - & - & 0.04 & 0.1 \\
\hline \multirow{2}{*}{$\begin{array}{l}\text { Final CPEs } \\
\text { manifestation }\end{array}$} & c.u. & - & 10.77 & 16.85 & 15.72 & 10.74 & 3.34 & 3.13 & \multirow[t]{2}{*}{60.55} & \multirow[t]{2}{*}{100} \\
\hline & $\%$ & - & 17.78 & 27.83 & 25.96 & 17.74 & 5.52 & 5.17 & & \\
\hline
\end{tabular}

As a result of the analysis, there were no measures of other state programs that contribute to the implementation of task 1 of the key state program - improving the regulatory framework controlling SMB sector. Consequently, CPEs in the sphere of SMB legal regulation improvement in the Kurgan region are not being formed. In relation to task 2 of the key state program - development and accessibility provisions of SMB support infrastructure - 6 out of 7 groups of activities participate in the formation of CPEs, which is $86 \%$ of the groups. When implementing this task, all CPEs groups are formed, except for others. At the same time, the most expressed are financial, infrastructure, and information and consulting effects. 3 out of 7 groups of measures, which is $43 \%$ of the groups, participate in the CPEs formation in relation to task 3 of the key state program - increasing the availability of financial resources for $S M B$. In particular, when implementing this task, financial, innovative, consulting, information and infrastructure CPEs are formed, while financial effects show a significant advantage over the rest. In relation to task 4 of the key state program - improving the mechanisms of financial and credit support for SMB - 5 out of 7 groups of measures ( $71 \%$ of groups) participate in the formation of CPEs. When implementing this task, financial, innovative, supervisory and licensing, consulting and information, and infrastructure CPEs are formed. At the same time, the group of financial effects is most expressed, while infrastructure effects are in second place with a significant gap from the first group. All 7 groups of measures contribute to the implementation of task 5 of the key state program - improving the competitiveness of SMBs, assisting them in promoting their products (works, services) and intellectual property results to the Russian Federation and foreign markets. This means that all the designated groups of CPEs are formed. At the same time, the most expressed are financial, marketing, and consulting and information effects. 3 out of 7 groups of measures (43\% of groups) participate in the formation of CPEs in the field of SMB in relation to task 6 of the key state program creation of conditions for improving the professional level of personnel for SMB. In particular, when implementing this task, innovative, information and consulting, infrastructure CPEs are formed. Among them the most expressed are information and consulting effects. In relation to task 7 of the key state program - promoting the development of innovative entrepreneurship - 6 out of 7 groups of activities $(86 \%$ of groups) participate in the formation of CPEs. When implementing this task, all CPEs 
groups are formed, except for others, and the most expressed are innovation and financial effects.

The largest number of state programs generates CPEs that contribute to the implementation of the task "Development and accessibility of SMB support infrastructure" - $65 \%$ of programs. By the number of state programs involved in the CPEs formation the tasks "Improving the SMB competitiveness, assisting them in promoting their goods (works, services) and results of intellectual activities on the Russian market and the markets of foreign countries" (59\% of programs) and "Improving the mechanisms of financial-credit support of SMB" (47\% of government programs) are also highlighted.

In the Kurgan region, the manifestation of financial CPEs in the implementation of SMB development tasks is higher than in other groups of CPEs (71\%). These effects are evident in the implementation of five of the seven tasks of the key state program aimed at the development of SMB, except for the tasks "Improving the regulatory framework controlling the sphere of SMB" and "Creating conditions for improving the professional level of personnel for SMB", which is $71 \%$ of the tasks. At the same time, the group of financial CPEs is most pronounced in four out of five implemented SMB development tasks. $29 \%$ of state programs and $28 \%$ of measures that create CPEs in the studied area contribute to the manifestation of this group of CPEs. Information and consulting CPEs $(10 \%)$ are in second place by the level of manifestation, formed by the implementation of $41 \%$ of government programs and $15 \%$ of activities that affect SMB development. This group of CPEs is shown in $86 \%$ of SMB development tasks, leading in one of them, aimed at creation of conditions for improving the professional level of personnel for SMB. In third place in terms of performance there is the group of infrastructure CPEs $(8 \%)$, which is provided by $41 \%$ of government programs and $13 \%$ of activities. This group of effects is reflected in $86 \%$ of SMB development tasks.

The remaining groups of CPEs (sales, innovation, supervisory and licensing, and others) are shown at the level of $0.1 \%$ to $5.3 \%$ and are provided by the implementation of $17-29 \%$ of state programs and $2-11 \%$ of activities. These groups are shown in $14-86 \%$ of the tasks, not being in the leading positions in any of them, except for innovative CPEs, the manifestation of which is most pronounced in the task "promoting the development of innovative entrepreneurship".

\section{Conclusions}

The results of the assessment of the overall level of CPEs manifestation in the implementation of the tasks of the key SMB development program allow to draw the following conclusions:

- the highest level of overall CPEs manifestation is achieved when implementing the tasks of increasing the availability of financial resources (28\%) and improving the mechanisms of financial and credit support for SMB (26\%);

- at the level of $18 \%$, total CPEs are shown when implementing the tasks of developing and ensuring the availability of infrastructure to support SMB and increasing SMB competitiveness, assisting them in promoting their products (works, services) and intellectual property results to the market of the Russian Federation and foreign markets; these effects are formed when implementing 59\% of programs and 55-59\% of activities;

- insignificant overall performance of CPEs at the level of 5-6\% is demonstrated during the implementation of tasks to create conditions for improving the professional level of personnel for SMB and promote the development of innovative entrepreneurship;

- there are no CPEs for the task on improving the regulatory framework governing the $S M B$ sector. 
The largest contribution to the achievement of the objectives of the SMB development program of the Kurgan region is made by the state programs "Development of the agroindustrial complex in the Kurgan region for 2014-2020", "Development of science and technology for the period up to 2020" and "Development of industry and increasing its competitiveness".

It is noteworthy that not all measures that affect SMB are linked to the objectives of the key state program. Thus, out of nine measures of the state program "Improvement of labor conditions and safety in the Kurgan region" and out of two measures of the state program "Information society" only one measure in each program is related to the tasks of the key state program. Activities of the state programs "Improving road safety in the Kurgan region", "Development of highways", "Management of state property and land resources of the Kurgan region", previously identified as affecting SMB, do not have links with the tasks of the key state program.

Thus, in the Kurgan region, the following state programs contribute to the greatest manifestation of CPEs in the SMB sector of the region:

- "Development of the agro-industrial complex in the Kurgan region for 2014-2020" ( $54 \%$ of cross-program effects);

- "Development of science and technology for the period up to 2020" (13\% of crossprogram effects);

- "Development of industry and increasing its competitiveness" (9\% of cross-program effects);

- The state program of the Kurgan region aimed at creation of favorable conditions for attracting investment in the economy ( $8 \%$ of cross-program effects);

- "Development of tourism in the Kurgan region" for 2014-2019 (7\% of cross-program effects).

The analysis carried out showed that the CPEs are forming in the region in the field of SMB. The degree of their manifestation depends on the number and strength of links between the activities of other state programs in the region and the tasks of the key state program.

\section{References}

1. T.S. Khudyakova, S.V. Doroshenko, Upravlenets (The Manager), 10 (2), 41-48 (2019)

2. S.N. Rukina, V.N. Samodurova, Accounting and statistics, 1 (45), 109-119 (2017)

3. A.G. Breusova, Bulletin of OmSU. Series: Economics, 2, 128-136 (2015)

4. N.S. Shmigol, Economy. Taxes. Law, 5, 114-125 (2017)

5. Assessment and determination of cross-influence of the main activities and departmental target programs, subprograms of one state programs of the Russian Federation on the goals and objectives of other state programs of the Russian Federation, 15 (FBSI Institute of macroeconomic research, 2013)

6. N.N. Shash, Upravlenets (The Manager), 1 (53), 4-15 (2015)

7. I. Ka Wai Lai, International Journal of Hospitality Management, 48, 52-67 (2015)

8. N. Menck, C. Weidig, J.C. Aurich, Approach for predicting production scenarios focused on cross impact analysis, Proceedings of the 47th CIRP Conference on Manufacturing Systems, 493-498 (2014)

9. S.V. Doroshenko, E.A. Trushkova, T.S. Khudyakova, Journal of economic theory, 15 (3), 420-428 (2018)

10. V.A Blaginin, E.L Plisetsky, Y.N Shedko, I.S Kobersy, NK Vasilieva, Int J Appl Bus Econ Res, 15 (23), 463-73 (2017) 